\title{
The CONTOUR Discovery Mission
}

\author{
Edward Reynolds, Mary Chiu, Robert Farquhar, David Dunham \\ Applied Physics Laboratory, The Johns Hopkins University \\ Laurel, MD 20723 \\ 443-778-5101 \\ ed.reynolds@jhuapl.edu
}

\begin{abstract}
Over a six-year period, CONTOUR will fly within 100 kilometers of three comet nuclei: Encke in 2003, Schwassmann-Wachmann-3 in 2006, and finally d'Arrest in 2008. CONTOUR will take images, make spectral maps, and analyze dust and gas. The payload includes fixed and tracking imagers that will provide high-resolution images and spectral maps. CONTOUR uses several innovative techniques for performing deep space missions at low cost. These include an indirect launch strategy, unattended hibernation, and an innovative mission and spacecraft design. This paper compares the CONTOUR design and development approach with other recent programs and identifies challenges CONTOUR will face in this era of faster, cheaper, and better.
\end{abstract}

\section{TABLE OF CONTENTS}

1. INTRODUCTION

2. CONTOUR MISSION DESCRIPTION

3. SIMPLIFIED SPACECRAFT DESIGN

4. MISSION PROFILE

5. INDIRECT LAUNCH STRATEGY

6. EARTH RETURN TRAJECTORIES

7. UNATTENDED HIBERNATION

8. CONCLUSIONS

9. REFERENCES

10. BIOGRAPHY

\section{INTRODUCTION}

NASA's approach to deep space exploration today is much different from ten or twenty years ago. In the seventies and eighties, there were just a few deep space missions. These missions used large, fully instrumented platforms to perform comprehensive science programs: Voyager, Galileo, Viking, etc. This approach suffered for several reasons. The rarity of launches was a source of tremendous pressure to add instruments and at the same time placed a lot of public attention on those few launches. Failure was not allowed, at any cost, since the entire space program and national prestige would be hurt. The additional instruments and the requirement of mission success resulted in extremely expensive programs.

U.S. Government work not protected by U.S. copyright.
The NASA of today is the NASA of faster, cheaper, and better. It represents a philosophical change in mission implementation in which mission success is measured across all of its missions. Instead of a few flagship programs, NASA's approach is to 'blacken the sky' with small, inexpensive, moderate-risk spacecraft in which each mission pursues a focused science goal. With such an approach, an occasional mission can fail without jeopardizing NASA's entire space science program. The acceptance of moderate risk enables programs that would be impossible if failure was not allowed. Mars Pathfinder demonstrated that there were less expensive approaches to landing on Mars than the risk overt approach taken by the Viking mission.

"Faster, cheaper, and better" became the NASA mantra of the 1990's. It became reality with the launches of Near Earth Asteroid Rendezvous (NEAR) and Mars Pathfinder in 1996. Both spacecraft were developed under NASA's Discovery program, in which small, moderate risk spacecraft were tasked to do billion dollar missions, at one quarter of the cost. Since then, the Discovery program has become a stable, long-term series of missions: Lunar Prospector (Discovery 3), StarDust (Discovery 4), Genesis (Discovery 5), and CONTOUR (Discovery 6). CONTOUR represents the second Discovery mission for the Applied Physics Laboratory (APL). NEAR was the first. APL learned a lot from building and operating NEAR over the last three years. In several ways CONTOUR and NEAR are similar. They both use similar components, construction, and qualification techniques. However, in other ways, CONTOUR goes one step beyond NEAR in this era of building and operating spacecraft with less and less money.

\section{Contour MisSION DESCRIPTION}

The Comet Nucleus Tour mission, or CONTOUR mission, will investigate several comet nuclei and assess their similarity and diversity. The CONTOUR mission concentrates on improving knowledge of key comet characteristics, especially the nucleus structure, composition, and its processes. Measurements will be obtained by a four-instrument payload: dual imagers, mapping spectrometer, dust analyzer, and mass spectrometer. The data these instruments collect will allow a meaningful assessment of the diversity of the nuclei of short-period comets. Table 1 identifies the mission's baseline science objectives. 
Table 1. CONTOUR Science objectives.

- Image parts of the nucleus at resolutions of 4 meters per pixel (25 times better than Giotto [1]) to reveal details of morphology and processes that will show us how comets work.

- Determine nucleus size, shape, rotation state, albedo/color heterogeneity, and activity through global imaging ( 100 to 500 meters per pixel).

- Map composition of nucleus surface and coma through spectroscopy.

- Obtain detailed compositional measurements of gas and dust in the near-nucleus environment at precisions comparable to those of Giotto or better.

- Assess level of outgassing through imaging, spectroscopy, gas and dust measurements.

- Assess the diversity of comets.

- If possible, investigate a new comet.

Comets are widely believed to be the most pristine, primitive bodies remaining in our solar system. Due to their pristine nature, comets should preserve clues to the important chemical and physical processes that took place when the planets formed, clues which have been so strongly altered by subsequent processes in larger bodies as to be difficult to recognize. Comet nuclei may be the only accessible objects that still retain a clear memory of the key chemical and physical events that took place during the formation of the solar system, processes such as condensation, agglomeration, and mixing. They probably also contain clues to the chemistry that led to the formation of the complex organic molecules known to exist in comet nuclei. Indeed, they may have been the original source of organics from which life arose. It is also very likely that comets contain a variety of pre-solar system, interstellar grains. These grains may indicate the origin of solar nebula materials in the galaxy.

Not all comets are alike. Remote sensing data indicate that there are significant differences between short-period and long-period comets in terms of the composition and relative amounts of the volatile and refractory components. Differences in the nature of gas emission among short-period comets and significant differences in gas-to-dust ratios have also been established. It is essential to understand to what degree these differences are due to primordial variations and to what degree they result from differences in subsequent evolutionary processes.

CONTOUR is an important first step in addressing these important issues. First, the CONTOUR mission will obtain detailed comparative data on three diverse short-period comets, allowing us to address the similarity and diversity of the gas and dust compositions. Second, the data obtained will be easily comparable to Halley Giotto data, helping us to address the question of diversity among Halley-like comets and truly short-period comets such as Encke, Schwassmann-Wachmann-3, and d'Arrest. Third, our dust analysis data will be directly comparable with those of the Stardust mission [2] (same dust analyzer), making it easier to extend the knowledge eventually gained from the direct analysis of comet Wild-2 sample materials to the CONTOUR comets. Finally, the mission flexibility of CONTOUR's trajectory allows the spacecraft to be retargeted in-flight to a new comet if one should appear. Based on a Monte-Carlo analysis, CONTOUR has a 98 percent chance of being able to intercept a new comet during its baseline mission [3]. Such an opportunity would address the most important question of diversity between short-period and long-period comets.

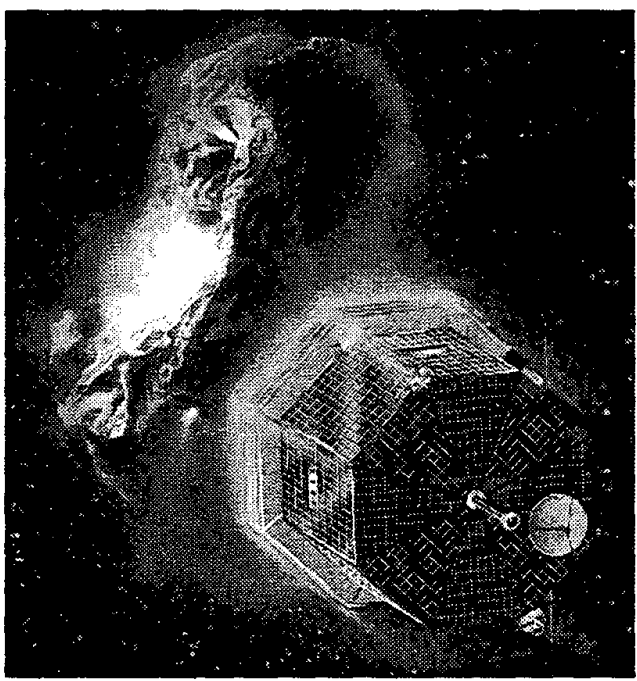

Figure 1. CONTOUR approaching comet nucleus.

The CONTOUR mission includes the investigation of three diverse short-period comets, including Encke. It is the next logical step in the exploration of comets beyond the flybys of Comet Halley, and is a valuable precursor to more complex comet missions. The three targets were selected because of their different physical characteristics and their close proximity to Earth around the encounter dates.

\section{SIMPLIFIED SPACECRAFT DESIGN}

CONTOUR represents JHU/APL's second generation of 'faster, cheaper, and better' deep space missions. NEAR [4] was the first generation. NEAR's approach to faster, cheaper, and better was to design and fabricate a flight system to perform a very focused mission. The system layout of NEAR took advantage of its mission deign geometry and operations approach. Over its mission trajectory, the Sun and Earth never really stray more than $30^{\circ}$ from each other. This allowed for the fixed solar array to be co-aligned with a fixed high gain antenna. NEAR was built using a traditional box-and-harness architecture in which subsystems are built up from discrete components that are cabled together.

CONTOUR continues NEAR's legacy of focused science and simple system geometry, but adds a strong commitment to design reuse and higher levels of functional integration to 
minimize the flight hardware. Design reuse comes at several levels and from several different flight programs. Figure 2 shows a top-level block diagram of the CONTOUR flight system. Figure 3 shows the spacecraft in its flight configuration. CONTOUR has few articulated mechanisms; the solar array is body mounted and does not require drive motors. The mission geometry allows CONTOUR to use fixed antennas. With the exception of a tracking mirror in the imager/ spectrograph (CRISP) instrument, all instrument and antenna pointing is controlled by moving the spacecraft.

The overall avionics architecture is derived from NASA's TIMED [5] program. It is based on the 'Integrated Electronics Module', or IEM. The IEM places a majority of a spacecraft's core avionics onto circuit cards that integrate into a chassis. The cards communicate with each other over a PCI backplane instead of a wire harness. The IEM philosophy is to have an open architecture in which card designs are reused (plug and play) to meet a specific mission's requirements. CONTOUR will be the test as to whether this philosophy to spacecraft construction works. The CONTOUR IEM will contain nine cards of which six cards are derived from TIMED. IEM functions include: the command system, data formatting system, data recorder, guidance and control processor, $\mathrm{X}$-band receiver, and $\mathrm{X}$-band transmitter. There are two IEM's for redundancy.

Other levels of design reuse are the use of subsystems from previous APL programs and the use of off-the-shelf components. CONTOUR's power system is derived from NEAR with little modification. The RF power amplifiers, stellar sensors, inertial reference units, battery and spinning sun sensors are existing designs already in production for other deep space programs.

The CONTOUR spacecraft has two main operating modes: hibernation and encounter mode. In hibernation mode, the spacecraft spins about its main axis at a spin rate of $20 \mathrm{rpm}$ and the spacecraft sleeps unattended for long periods of time. Encounter mode is 3-axis stabilized, which is required for the imager operation. The spacecraft attitude during an encounter is fixed with the dust shields and instruments aligned to the comet relative vector with only a small roll maneuver to keep the nucleus centered within the tracking imager. During an encounter, the spacecraft is protected by its dust shield system mounted in the ram direction. The shield design is based on the Nextel multi-shock shield concept developed by NASA for Space Station [6]. The basic concept of the shield is to use multiple layers of high-density fiber fabrics (Nextel) separated by space to break up an impacting dust particle and then to absorb the disbursed particle pieces using a Kevlar backstop.

For communications with Earth, CONTOUR will use a new two-way non-coherent doppler technique instead of deep space transponders [9]. With the new method, normal receivers and transmitters are used. The uplink Doppler shift is measured onboard the spacecraft and embedded (as N1, N2 counts) in the downlink telemetry frame. The downlinked doppler shift is measured using the traditional method by the Deep Space Network. The N1 and N2 counts are applied to the measured doppler to null out any oscillator errors from the spacecraft. This method has been shown to produce performance equal to a deep space transponder without the complexity and cost of a coherent system [10].

\section{CONTOUR Avionics Architecture}

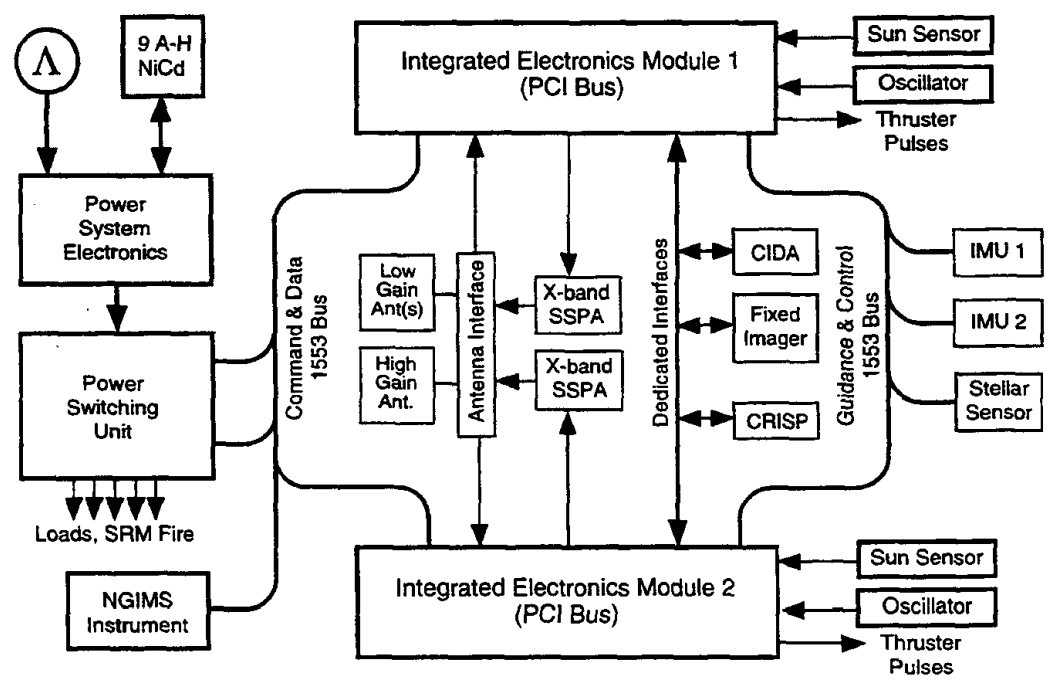

Figure 2 - CONTOUR Top Level System Block Diagram. 
The payload consists of four instruments: a wide-angle imager, a tracking high-resolution imager and spectral mapper (CRISP), a dust analyzer (CIDA), and a neutral gas/ion mass spectrometer (NGIMS). The core of the CONTOUR imaging and spectral mapping experiments is CRISP. The imager/spectral mapper will have a target tracking capability to image the nucleus from multiple directions. The wideangle camera to serve as backup and provide global views of the nucleus and coma. The dust analyzer is a copy of the instrument being supplied for the Stardust mission. It will be built and calibrated by vonHoerner and Sulger of Germany. NGIMS will be provided by NASA GSFC.

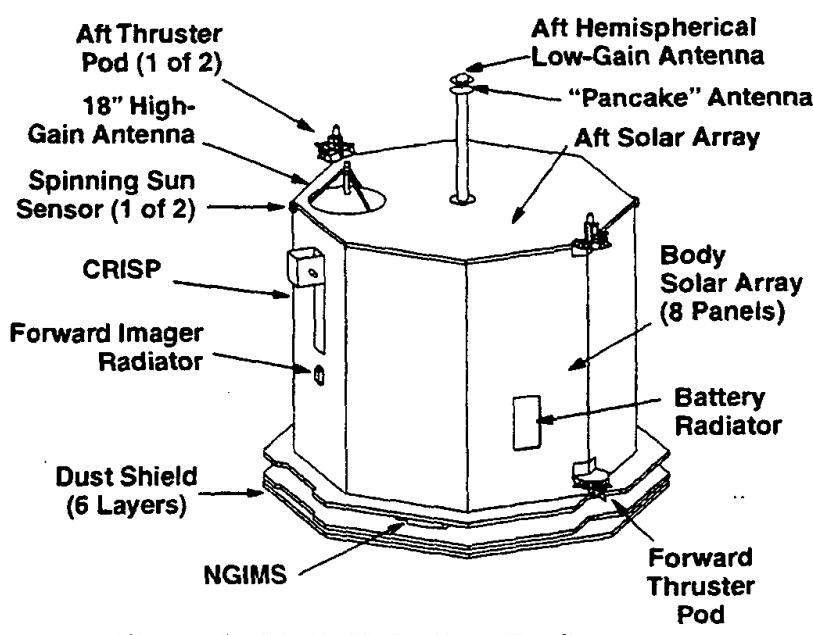

Figure 3. CONTOUR Flight Configuration

\section{Mission Profile}

The CONTOUR mission uses Earth-gravity assist maneuvers to accomplish the multiple encounters. The CONTOUR mission profile is extremely flexible and could be modified to include a first-ever study of a new comet. Six swingbys are used to accomplish the three comet encounters over a 6year period [8].

A. bipolar plot of the CONTOUR trajectory sequence is shown in Fig. 4. Table 2 provides key data regarding CONTOUR's baseline mission. The sequence begins with a 1 year Earth-return loop (segment 1) that positions the spacecraft for an encounter with comet Encke in November 2003. Three Earth-gravity assist maneuvers are then used to retarget the spacecraft for an encounter with comet Schwassmann Wachmann-3 in June 2006. Two more Earth gravity assists enable CONTOUR to reach its final target, comet d'Arrest, in August 2008. Throughout the 6-year mission, the spacecraft-Earth distance is always less than 0.70 Astronomical Units (AU). One $A U$ is the distance between Earth and the Sun. The spacecraft-Sun distance varies between 0.83 and $1.35 \mathrm{AU}$. Especially noteworthy is the fact that all three encounters are extremely close to the Earth $(0.27,0.33$, and $0.36 \mathrm{AU})$. All baseline comet encounters take place at times of excellent viewing from Earth, providing opportunities for cooperative worldwide supporting observations by ground-based and Earth-orbiting telescopes. In addition to professional astronomers, student and amateur observing teams are expected to play an important role in conducting these supporting observations.

\begin{tabular}{|c|c|}
\hline Earth-Swingby Maneuvers \\
\hline Date & $\begin{array}{c}\text { Perigee } \\
\text { (Earth radii) }\end{array}$ \\
\hline $8-13-2003$ & 13.2 \\
$8-13-2004$ & 6.4 \\
$2-09-2005$ & 9.3 \\
$2-09-2006$ & 2.9 \\
$2-09-2007$ & 4.0 \\
$2-10-2008$ & 1.1 \\
\hline
\end{tabular}

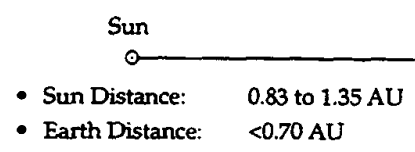

6-month segment (3) between 8-13-04 and 2-9-05 is just a point at the Earth in this diagram.

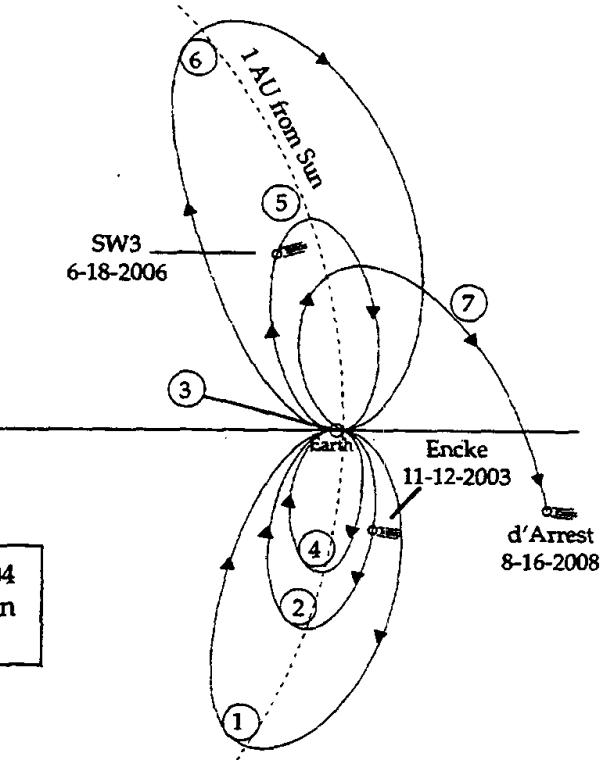

Figure 4 - Bipolar Plot of CONTOUR Trajectory Sequence. The plot traces the spacecraft trajectory with respect to a fixed Sun-Earth line (Ecliptic-plane view). 
Table 2. CONTOUR Baseline Mission Profile

\begin{tabular}{|c|c|c|c|c|}
\hline$\underset{8-03}{\infty} \underset{11-\infty}{\infty}$ & $\underset{204}{\oplus} \rightarrow-05$ & $\rightarrow \underset{206}{ } \rightarrow \underset{8-06}{\delta}$ & $\rightarrow \underset{207}{\oplus}$ & 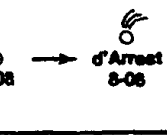 \\
\hline \multicolumn{5}{|c|}{ Earth-Swingby Manouvere } \\
\hline \multirow{2}{*}{ Swingby Date } & \multirow{2}{*}{$\begin{array}{c}\text { Perlgee } \\
\text { (Earth Radil) }\end{array}$} & \multicolumn{3}{|c|}{ Hollocentric Orbit After Swingby } \\
\hline & & Perihelion (AU) & Aphelion (AU) & Incl. (Degrees) \\
\hline Aug. 13, 2004 & 6.43 & 0.98 & 1.02 & 12.0 \\
\hline Feb. 9, 2005 & 9.33 & 0.93 & 1.07 & 11.5 \\
\hline Feb. 9, 2006 & 2.87 & 0.92 & 1.08 & 10.9 \\
\hline Feb. 9, 2007 & 4.01 & 0.83 & 1.17 & 6.4 \\
\hline Feb. 10,2008 & 1.10 & 0.93 & 1.48 & 6.5 \\
\hline \multicolumn{5}{|c|}{ Comot Encounters } \\
\hline Encounter Dato & $\begin{array}{l}\text { Sun Diatance } \\
\text { (AU) }\end{array}$ & $\begin{array}{l}\text { Earth Distance } \\
\text { (AU) }\end{array}$ & $\begin{array}{l}\text { Phase Angle } \\
\text { (Degrees) }\end{array}$ & $\begin{array}{l}\text { Fhyby Speed } \\
\text { (kn/sec) }\end{array}$ \\
\hline Enoke: 11-13-03 & 1.07 & 0.27 & 12 & 28.2 \\
\hline SW3: 6-18-06 & 0.95 & 0.33 & 100 & 14.0 \\
\hline d'Amest: 8-16-08 & 1.35 & 0.36 & 68 & 11.8 \\
\hline
\end{tabular}

During the Earth-gravity assist that precedes each of the comet encounters, the spacecraft and its instruments are thoroughly checked. Using tracking data, the spacecraft's trajectory is accurately determined, and a coarse targeting maneuver is executed 25 days prior to encounter. The 'miss' distance is baselined at $100 \mathrm{~km}$ from the nucleus. This distance is a trade-off between science value and spacecraft safety. For CONTOUR, at $100 \mathrm{~km}$ there is less than a one percent chance of encountering dust grains of a size that could damage the spacecraft [12]. Earth-based optical and radar observations of the comet's dust environment taken shortly before the encounter will be used to update the miss distance. Imaging and spectral observations begin ten days prior to encounter when the imaging resolution is about 200 $\mathrm{km} /$ pixel. Optical Navigation (OpNav) and coma monitoring observations will be obtained each day. The final targeting burn is executed one day before closest approach. The spacecraft will remain on a velocity vector orientation, allowing continuous coverage by the gas and dust instruments.

\section{INDIRECT LAUNCh STRATEgY}

Table 3 summarizes the CONTOUR launch plan. To lower launch costs, CONTOUR will use a new technique called 'indirect launch mode', or ILM [7]. ILM has a number of important advantages over a conventional direct launch including:
- a significant gain in delivered spacecraft weight,

- an unconstrained launch window,

- spacecraft checkout while in Earth orbit,

- choosing injection time to optimize trajectory,

- efficient correction of launch dispersion errors.

Table 3. CONTOUR Baseline Launch Summary.

\begin{tabular}{|l|l|}
\hline Launch window & June 26 to July 31, 2002 \\
\hline $\begin{array}{l}\text { Launch-energy } \\
\text { requirement }\end{array}$ & $\mathrm{C}_{3} \leq-9.8 \mathrm{~km}^{2} / \mathrm{sec}^{2}$ \\
\hline Launch vehicle & $\begin{array}{l}\text { Delta } 7425-9.5 \text { (99\% PCS, } \\
3712 \mathrm{C} \text { attachment fitting) }\end{array}$ \\
\hline $\begin{array}{l}\text { Allowable launch } \\
\text { mass }\end{array}$ & $\mathrm{M}_{0}=1005 \mathrm{~kg}$ \\
\hline $\begin{array}{l}\text { - Spacecraft placed into high-apogee (11.7 Earth radii) } \\
\text { phasing orbit (period 0.95 days) }\end{array}$ \\
\hline $\begin{array}{l}\text { - STAR-30 solid rocket motor used to place spacecraft } \\
\text { into one-year Earth-return trajectory on August 13, 2002. }\end{array}$ \\
\hline $\begin{array}{l}\text { - Am Earth gravity-assist maneuver in August 2003 tar- } \\
\text { gets the spacecraft toward an encounter with comet En- } \\
\text { cke in November 2003. }\end{array}$ \\
\hline
\end{tabular}

PCS = probability of commanded shutdown

The mission scenario requires injection at a fixed time on August 13,2002 . Instead of relying on the launch vehicle to 
place the spacecraft directly onto its interplanetary trajectory, the rocket inserts the spacecraft into a highly elliptical 'phasing orbit' as shown in Figure 5. The apogee for the phasing orbits is baselined at 11.7 Earth radii, with corresponding period of 0.95 days. With phasing orbits, perigee heights and inclinations can be adjusted with small delta-V corrections to satisfy required conditions for the injection maneuver on August 13,2002. The injection maneuver is performed with a STAR-30 solid rocket motor at a perigee altitude of approximately $300-\mathrm{km}$. The delta-V cost is 2,057 $\mathrm{m} / \mathrm{s}$. The use of phasing orbits makes it possible to schedule a launch anytime before July 31,2002 . This flexibility allows considerable freedom in specifying a launch window for CONTOUR. This approach essentially relieves the launch vehicle of the very tight launch window constraints launch can occur at any time prior to the time necessary to complete a minimum number of phasing orbits (typically three). CONTOUR plans to use a 36-day launch window.

CONTOUR will use a Delta-7425 vehicle (four strap-on motors and a STAR-48 third stage) for insertion into a phasing orbit $\left(\mathrm{C}_{3}=-9.8 \mathrm{~km}^{2} / \mathrm{sec}^{2}\right)$ with an apogee of 11.7 Earth radii. The Delta-7425 can deliver a mass of about $1,005-\mathrm{kg}$ to this orbit, of which $452-\mathrm{kg}$ is spacecraft $(553-\mathrm{kg}$ is solid rocket fuel). From this orbit, the solid rocket accel- erates the spacecraft to a $\mathrm{C}_{3}$ of $37.5 \mathrm{~km}^{2} / \mathrm{sec}^{2}$. If CONTOUR uses a direct launch, the spacecraft mass must be reduced to $280-\mathrm{kg}$ using the same class launch vehicle. Moreover, by using the ILM, the post launch deterministic delta-V is zero. If a direct launch approach were used, the post launch deterministic delta-V would increase from 0 (ILM case) to 600 $\mathrm{m} / \mathrm{sec}$ ! Without the ILM, the CONTOUR mission would not have been possible unless a larger, more expensive launch vehicle was used.

NEAR's trajectory required a launch energy of 25.8 $\mathrm{km}^{2} / \mathrm{sec}^{2}$ - substantially less than CONTOUR's (37.5 $\mathrm{km}^{2} / \mathrm{sec}^{2}$ ). The NEAR launch used the traditional direct launch method using a Delta-7925 (adds five additional strap-on motors to the 7425 rocket used by CONTOUR). To be fair, it should be noted that NEAR's large delta rocket is delivering more mass onto its final trajectory than CONTOUR's concept using ILM and a small Delta rocket: 805$\mathrm{kg}$ versus $452-\mathrm{kg}$. But if NEAR had used the ILM, the spacecraft mass placed onto the final trajectory would have increased by $110-\mathrm{kg}$ [7] and the fuel required for deterministic delta- $V$ would have decreased substantially. NEAR would have required a drastically different system layout to accommodate a solid rocket motor.

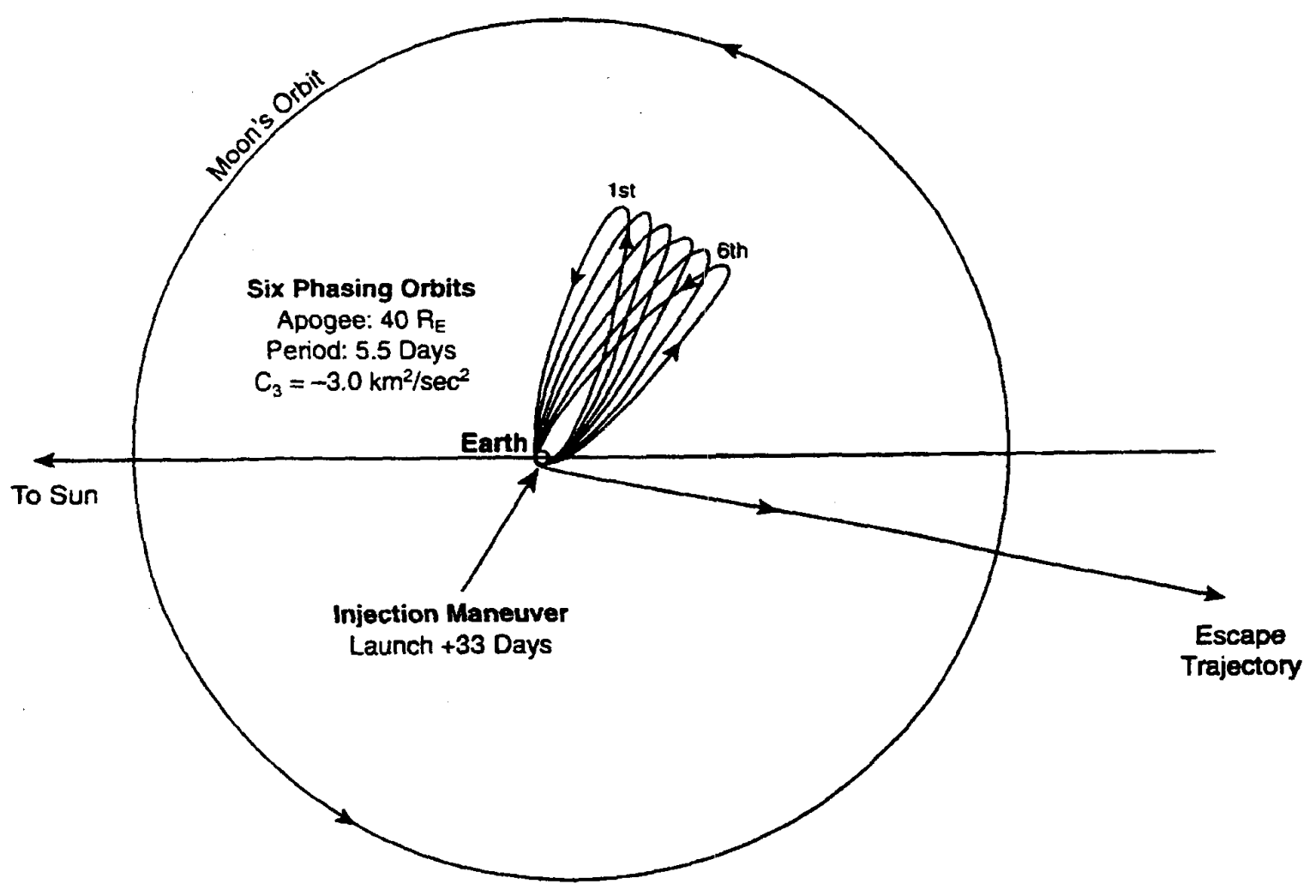

Figure 5. Generic example of an ILM launch sequence. 


\section{USE OF EARTH RETURN TRAJECTORIES}

The simplicity of the CONTOUR mission design is the use of Earth return trajectories and Earth gravity assist maneuvers to sequence from one comet encounter to the next. CONTOUR uses a total of six Earth-renum loops/gravityassist maneuvers [6]. Three of the trajectory loops contain comet encounters. The other three loops do not involve encounters and are used for time phasing. This approach greatly reduces the amount of onboard propellant required. Earth return trajectories for multi-comet encounters was first proposed in 1973 [6] for a dual comet mission (that proposal was not funded). Earth return/gravity assist was first successfully used to extend the Giotto mission. After Giotto's encounter with comet Halley in March 1986, a small propulsive maneuver was used to return the spacecraft to Earth for a gravity-assist maneuver to successfully intercept Comet Grigg-Skjellerup in 1992. Without the use of the Earth return trajectory and the gravity assist maneuvers, the amount of fuel required would be prohibitive and three dedicated spacecraft would be required to complete the baseline mission.

A very powerful feature of using Earth return trajectories and gravity assist maneuvers is its flexibility to retarget the spacecraft to other flyby targets, such as a newly discovered comet. Any of the Earth encounters following the Encke encounter in 2003 could be used to re-direct CONTOUR to a new comet if it were detected early enough. Statistical Monte Carlo studies have shown that CONTOUR has a $98 \%$ probability of being capable of retargeting to a new comet between 2004 and 2008.

The mission flexibility also means that CONTOUR can easily extend its mission to other known comets with very little extra delta-V. Several different extended mission concepts have been evaluated. The reachable target comets include: Tempel-2, Giacobini-Zinner, Grigg-Skjellerup, and Encke again.

\section{UNATTENDED HibERNATION}

Perhaps the largest improvement in reducing mission costs is to reduce a mission's reliance on a fully staffed mission operations team and to reduce the need for regular communication with the spacecraft throughout the mission. NASA's deep space missions rely on three globally separated stations of 70-meter and 34-meter antennas for communication. These stations are located in Spain, Australia, and California and comprise the Deep Space Network, or DSN. A mission's DSN usage varies greatly depending on spacecraft activity. During periods of high activity the usage is nearly continuous. During periods when the spacecraft is cruising, maintenance contacts are limited to about one eight-hour pass per week. A large portion of a mission's operational costs is associated with DSN usage. The current DSN aperture fees vary between 560 (34-Meter) and 2,240 dollars (70-meter) (FY98\$) [9] per hour. By making the spacecraft more autonomous, the costs associated with routine contacts can be significantly reduced.

During the cruise intervals between comet encounters and Earth-gravity assist maneuvers, the CONTOUR spacecraft will be put to sleep in a spin-stabilized 'hibernation' mode (similar to the Giotto spacecraft from 1986 to 1990). The spacecraft will be oriented with its major axis normal to the orbit plane and then spin stabilized at $20 \mathrm{rpm}$. Instruments and many subsystems will be powered off. Only the command receivers, thermostatically controlled heaters, and critical core components will be powered. It is assumed that there will be no ground contact with the spacecraft while it is in this hibernation state. Over CONTOUR's mission lifetime of roughly 6 years, DSN assets are required just $6 \%$ of the time. In comparison, NEAR has no hibernation mode and remains active and three axis stabilized throughout its entire mission. It was not designed to cruise for long periods while unattended. Because of this, NEAR is contacted at least once per week (8-hour pass per contact) throughout its three-year cruise. NEAR has been fully staffed by a mission operations team since launch.

\section{Conclusions}

The first generation of faster, cheaper, better focused on designing, fabricating, and testing the flight system more quickly and less expensive than past deep space missions. These missions streamlined the development time from 5 to 10 years to just 27 months. With the reduction in development time, the development cost was reduced accordingly. With the second-generation missions, like CONTOUR, the focus is to reduce the operations costs and the launch costs. The approach is to make spacecraft more autonomous, by using very simple hibernation schemes. CONTOUR also benefits from creative techniques like ILM and gravityassists to reduce launch vehicle lift requirements and costs.

\section{REFERENCES}

[1] N. Calder, Giotto to the Comets, Presswork Press, 1992.

[2] Ken Atkins, STARDUST: Discovery's Interstellar Dust and Cometary Sample Return Mission, IEEE Aerospace Conference, 1997.

[3] A. Friedlander and D. German, "Probability Analysis of New Comet Flyby for CONTOUR Mission", Advances in the Astronautical Sciences, Vol. 97, 1998, pp. 1645-1663.

[4] A.G. Santo, S.C. Lee, and A.F. Cheng, Near Earth As- 
teroid Rendezvous (NEAR) spacecraft overview, Proc. IEEE Aeorspace Applications Conference, 131-144, Aspen, CO, 1996.

[5] G. Cameron and D. Kusnierkiewicz, Reducing the lifecycle cost of space systems, $49^{\text {th }}$ International Astronautical Congress, Melbourne, Australia, Sep 28 - Oct 2, 1998.

[6] E. Cristiansen, Design and Performance Equations for Advanced Meteoroid and Debris Shields, International Journal of Impact Engineering, Nov 1992.

[7] Robert W. Farquhar and David Dunham, "The Indirect Launch Mode: A New Launch Technique for Interplanetary Missions" 1998 IAA L98-0901, Date here.

[8] Robert W. Farquhar, "The Use of Earth-Return Trajectories For Missions to Comets", Fourth John V. Breakwell Memorial Lecture, IAF-98-A.5.01, $49^{\text {th }}$ International Astronautical Congress, Melbourne, Austrailia, September 28October 2, 1998.

[9] NASA Mission Operations and Communications Services, AO 98-OSS-XX, March 1998, Section 2.4.1.

[10] J. R. Jensen and R. S. Bokulic, "Accurate Doppler Navigation with a Simple Spacecraft Transceiver", 1999 IEEE Aerospace Conference, March 6-13, 1999.

[11] J. R. Jensen and R. S. Bokulic, "Results of Engineering Tests With the DSN to Evaluate Noncoherent Doppler Tracking performance", JHU/APL memo SRO-98M-16, July 28, 1998.

[12] Comet Nucleus Tour Feasibility Study, Dr. J. Veverka Principal Investigator, NASA Discovery Mission Step Two Selection, pgs 43-44, August 1997.

\section{BIOGRAPHY}

Edward Reynolds is a member of APL's Principal Professional Staff. He received a B.S. in electrical engineering from Virginia Tech in 1985. He has been a member of APL's technical staff since 1985 . His past experience includes system engineer of the Pre-Phase A feasibility study of the NEAR mission, and as system engineer for the CONTOUR feasibility study. Mr. Reynolds is the Mission System Engineer for the CONTOUR flight program.
Mary Chiu is a member of APL's Principal Professional Staff. She received a B.S. in physics from University of Toledo in 1974. She also received a M.S. in applied physics in 1979 from the Johns Hopkins University. She has been a member of APL's technical staff since 1975. Her responsibilities at APL have included program management for several spacecraft instruments and Program Manager for NASA's ACE spacecraft, which launched in 1997. Currently, Ms. Chiu is the Project Manager for the CONTOUR flight program.

Robert W. Farquhar is a member of APL's Principal Professional Staff. He received a Ph.D. in astronautical sciences from Stanford University in 1969. Before coming to APL, Dr. Farquhar worked at several NASA facilities including Goddard Space Flight Center and NASA Headquarters. Since coming to APL in 1990, he has served as mission director for the NEAR program and will serve in the same role for CONTOUR. The indirect launch mode is one of Dr. Farquhar's concepts and is currently under patent review. The use of Earth return trajectories and capitalizing from gravity assist maneuvers is another trademark of Dr. Farquhar's mission design teclanique.

David Dunham is a member of APL's Senior Professional Staff. Dr. Dunham has an extensive background in astrodynamics, spacecraft trajectory design, astronomy, and computer programming. His experience includes the design of numerous complex interplanetary and translunar trajectories for several,including NEAR, International Cometary Explorer, SOHO, and Wind. He is president of the International Occultation Timing Association. He received a B.A. Astronomy from University of California, Berkeley in 1964 and a Ph.D. Astronomy from Yale University in 1971.
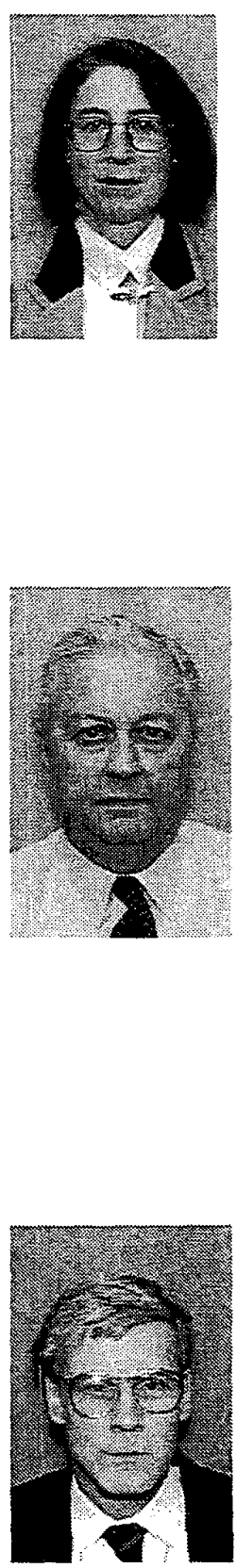